Bull. Austral. Math. Soc.

Vol. 37 (1988) [337-343]

\title{
FOURTH-ORDER BOUNDARY VALUE PROBLEMS AT NONRESONANCE
}

\author{
YISONG YANG
}

\begin{abstract}
We establish under nonuniform nonresonance conditions an existence and uniqueness theorem for a linear, and the solvability for a nonlinear, fourth-order boundary value problem which occurs frequently in plate deflection theory.
\end{abstract}

\section{INTRODUCTION}

The linear fourth-order boundary value problem

$$
\begin{gathered}
d^{4} y / d x^{4}-f(x) y=g(x), \quad 0<x<1, \\
y(0)=y_{0}, \quad y(1)=y_{1}, \quad y^{\prime \prime}(0)=\tilde{y}_{0}, \quad y^{\prime \prime}(1)=\tilde{y}_{1}
\end{gathered}
$$

and its nonlinear version

$$
\begin{gathered}
d^{4} y / d x^{4}-F\left(x, y, y^{\prime}, y^{\prime \prime}, y^{\prime \prime \prime}\right) y=G\left(x, y, y^{\prime}, y^{\prime \prime}, y^{\prime \prime \prime}\right), \quad 0<x<1, \\
y(0)=y_{0}, \quad y(1)=y_{1}, \quad y^{\prime \prime}(0)=\bar{y}_{0}, \quad y^{\prime \prime}(1)=\bar{y}_{1}
\end{gathered}
$$

occur frequently in plate deflection theory. Usmani [4] states an existence and uniqueness theorem for problem (1) under the condition $f(x)<\pi^{4}$ and in a recent communication [5] we observe that the existence and uniqueness theorem for problem (1) holds under the general condition $f(x) \neq k^{4} \pi^{4}$ for $k=1,2, \ldots$. This last condition restricts the problem to the so-called uniform nonresonance case. In Section 2 we establish an existence and uniqueness theorem for problem (1) under a nonuniform nonresonance condition which allows some "partial" resonance, that is, the occurence of $f(x)=k^{4} \pi^{4}$ on a subset of $[0,1]$. In Section 3 we apply the theorem obtained in Section 2 to establish a solvability theorem for the nonlinear problem (2), also under a nonuniform nonresonance condition which improves some known results (for example, Aftabizadeh [1]). Our argument below is a combination of the Fredholm alternative theorem and a modification of the method developed by Nkashama and Willem [3]. Throughout this paper all functions are assumed to be real and continuous.

Received 14 July, 1987

Copyright Clearance Centre, Inc. Serial-fee code: 0004-9729/88 \$A2.00+0.00. 


\section{The LINEAR PROBLEM}

Let $\operatorname{Im}(F)$ denote the image of a function $F:[0,1] \rightarrow \mathbf{R}$ and $\operatorname{Int}(A)$ the interior of the set $A \subseteq \mathbf{R}$.

TheOREM 1. Suppose that $f(x)$ satisfies

$$
\begin{gathered}
f^{-1}\left(k^{4} \pi^{4}\right) \neq[0,1], \quad k=1,2, \ldots, \text { and } \\
\left\{k^{4} \pi^{4}: k=1,2, \ldots\right\} \cap \operatorname{Int}(\operatorname{Im}(f))=\emptyset .
\end{gathered}
$$

Then Problem (1) has a unique solution.

Remarks. (1) The uniform nonresonance condittion which can be stated as $\left\{k^{4} \pi^{4}: k\right.$ $=1,2, \ldots\} \cap \operatorname{Im}(f)=0$ satisfies conditions (3a) and (4a).

(2) Condition (3a) is in fact necessary for the uniqueness and existence of a solution to problem (1), and this condition can be restated as

$$
f(x) \not \equiv k^{4} \pi^{4}, \quad k=1,2, \ldots
$$

(3) Since, by the continuity of $f, \operatorname{Im}(f)$ is a closed interval, therefore condition (4a) is equivalent to the statement that either

$$
f(x) \leqslant \pi^{4} \text { for all } x \in[0,1]
$$

or there is some integer $k \geqslant 1$ such that

$$
k^{4} \pi^{4} \leqslant f(x) \leqslant(k+1)^{4} \pi^{4} \text { for all } x \in[0,1] .
$$

(4a) is thus a condition which allows some "partial" resonance.

Proof of Theorem 1: Let $G(x, s)$ be the Green function of the problem

$$
\begin{gathered}
u^{\prime \prime}(x)=h(x), \quad 0<x<1, \\
u(0)=u(1)=0 .
\end{gathered}
$$

Then we can convert Problem (1) into an integral equation over the space $C[0,1]$ :

$$
y-T y=z
$$

where

$$
\begin{gathered}
(T y)(x)=\int_{0}^{1} \int_{0}^{1} G(x, s) G(s, t) f(t) y(t) d t d s, \text { and } \\
z(x)=y_{0}+x\left(y_{1}-y_{0}\right)+\int_{0}^{1} G(x, s)\left[\bar{y}_{0}+s\left(\bar{y}_{1}-\tilde{y}_{0}\right)+\int_{0}^{1} G(s, t) g(t) d t\right] d s .
\end{gathered}
$$


Now it suffices to show that for any $z \in C[0,1]$, equation (5) is uniquely solvable in the space $C[0,1]$. Since $T: C[0,1] \rightarrow C[0,1]$ is a linear compact operator, by the well-known Fredholm alternatives (see, for example, Gilbarg and Trudinger [2, pi1]) we see that it will be enough to prove that the only solution of equation

$$
y-T y=0
$$

is the trivial solution $y=0$. We proceed as follows.

Convert equation (6) back into the boundary value problem

$$
\begin{gathered}
d^{4} y / d x^{4}-f(x) y=0, \quad 0<x<1 \\
y(0)=y(1)=y^{\prime \prime}(0)=y^{\prime \prime}(1)=0 .
\end{gathered}
$$

Since $\{\sqrt{2} \sin j \pi x: j=1,2, \ldots\}$ is a complete orthonormal basis of $L^{2}[0,1]$, we have, in $L^{2}[0,1]$,

$$
\begin{gathered}
y=\sqrt{2} \sum_{j=1}^{\infty} a_{j} \sin j \pi x \\
d^{4} y / d x^{4}=\sqrt{2} \sum_{j=1}^{\infty} a_{j} j^{4} \pi^{4} \sin j \pi x .
\end{gathered}
$$

Denote by $(.,$.$) the standard inner product defined on L^{2}[0,1]$. Then the selfadjointness of the operator $d^{4} / d x^{4}$ gives the relation

$$
\begin{aligned}
0 & =\left(d^{4} y / d x^{4}-f y, y_{2}-y_{1}\right) \\
& =\left(d^{4} y_{2} / d x^{4}-f y_{2}, y_{2}\right)-\left(d^{4} y_{1} / d x^{4}-f y_{1}, y_{1}\right)
\end{aligned}
$$

where the decomposition $y=y_{1}+y_{2}$ is made such that

$$
\begin{aligned}
& y_{1}=0, \quad y_{2}=y, \quad \text { if } f \text { satisfies }(4 \mathrm{~b}) ; \\
& y_{1}=\sqrt{2} \sum_{j=1}^{k} a_{j} \sin j \pi x \quad \text { and } \\
& y_{2}=\sqrt{2} \sum_{j=k+1}^{\infty} a_{j} \sin j \pi x \quad \text { if } f \text { satisfies }(4 \mathrm{c}) .
\end{aligned}
$$

If $f$ satisfies (4b), we have from (8) and the Parseval equality that

$$
\begin{aligned}
0 & =\left(d^{4} y / d x^{4}, y\right)-(f y, y) \\
& \geqslant \sum_{j=1}^{\infty} a_{j}^{2}\left(j^{4} \pi^{4}-\pi^{4}\right) .
\end{aligned}
$$


Therefore $a_{j}=0, j=2,3, \ldots$ Hence, $y=\sqrt{2} a_{1} \sin \pi x$. Inserting this into (7), we get

$$
a_{1}\left(\pi^{4}-f(x)\right) \sin \pi x=0, \quad \text { for all } x \in[0,1] \text {. }
$$

Now using (3b) we conclude that $a_{1}=0$, so $y=0$. If $f$ satisfies (4c), we have

$$
\begin{aligned}
\left(d^{4} y_{2} / d x^{4}-f y_{2}, y_{2}\right) & \geqslant\left(d^{4} y_{2} / d x^{4}, y_{2}\right)-(k+1)^{4} \pi^{4}\left(y_{2}, y_{2}\right) \\
& =\sum_{j=k+1}^{\infty} a_{j}^{2}\left(j^{4} \pi^{4}-(k+1)^{4} \pi^{4}\right) \geqslant 0
\end{aligned}
$$

and

$$
\begin{aligned}
\left(d^{4} y_{1} / d x^{4}-f y_{1}, y_{1}\right) & \leqslant\left(d^{4} y_{1} / d x^{4}, y_{1}\right)-k^{4} \pi^{4}\left(y_{1}, y_{1}\right) \\
& =\sum_{j=1}^{k} a_{j}^{2}\left(j^{4} \pi^{4}-k^{4} \pi^{4}\right) \leqslant 0 .
\end{aligned}
$$

Substituting the above two inequalities into (8) we obtain

$$
\begin{gathered}
\left(d^{4} y_{2} / d x^{4}-f y_{2}, y_{2}\right)=0 \\
\sum_{j=k+1}^{\infty} a_{j}^{2}\left(j^{4} \pi^{4}-k^{4} \pi^{4}\right)=0 \\
\left(d^{4} y_{1} / d x^{4}-f y_{1}, y_{1}\right)=0 \\
\sum_{j=1}^{k} a_{j}^{2}\left(j^{4} \pi^{4}-k^{4} \pi^{4}\right)=0
\end{gathered}
$$

Hence $a_{j}=0, j \neq k, k+1$. Consequently $y_{1}=\sqrt{2} a_{k} \sin k \pi x$ and $y_{2}=$ $\sqrt{2} a_{k+1} \sin (k+1) \pi x$. Inserting these expressions into (10a) and (9a) respectively and observing condition ( 3 ) we obtain $a_{k}=a_{k+1}=0$, so again $y=0$.

The proof of the theorem is now complete.

\section{The nonlinear pRoblem}

In this section we study the nonlinear problem (2). We use $X$ to denote an arbitary point in $\mathbf{R}^{4}$. First we formulate (3)-(4) type conditions for the function $F(x, X)$.

(H) Suppose that $F$ is a bounded function on $[0,1] \times \mathbf{R}^{4}$ and define $a(x), b(x) \in$ $L^{\infty}[0,1]$ by

$$
a(x)=\inf _{X} F(x, X), \quad b(x)=\sup _{X} F(x, X),
$$

where the measurability of $a$ and $b$ is assumed. Assume further that either $b(x) \leqslant \pi^{4}$ a.e. or there is an integer $k$ such that $k^{4} \pi^{4} \leqslant a(x) \leqslant b(x) \leqslant(k+1)^{4} \pi^{4}$ a.e. and moreover, neither $a^{-1}\left(k^{4} \pi^{4}\right)$ nor $b^{-1}\left(k^{4} \pi^{4}\right)$ is a measure of 1 . 
THEOREM 2. If $G(x, X)$ is a bounded function and function $F(x, X)$ satisfies hypothesis (H), then Problem (2) has at least one solution.

Proof: The proof uses Theorem 1 and the Schauder fixed point theorem. Define a map $T: C^{3}[0,1] \rightarrow C^{3}[0,1]$ by $u=T w$, where $u, w$ are related by

$$
\begin{gathered}
d^{4} u / d x^{4}-F\left(x, w, w^{\prime}, w^{\prime \prime}, w^{\prime \prime \prime}\right) u=G\left(x, w, w^{\prime}, w^{\prime \prime}, w^{\prime \prime \prime}\right), \quad 0<x<1, \\
u(0)=y_{0}, \quad u(1)=y_{1}, \quad u^{\prime \prime}(0)=\bar{y}_{0}, \quad u^{\prime \prime}(1)=\bar{y}_{1} .
\end{gathered}
$$

We see easily that $f(x)=F\left(x, w(x), w^{\prime}(x), w^{\prime \prime}(x), w^{\prime \prime \prime}(x)\right)$ satisfies conditions $(3)$ and (4) so the map $T$ is well-defined. First we show that the image of $T, \operatorname{Im}(T)$ say, is a bounded subset of $C^{3}[0,1]$.

Otherwise if $\operatorname{Im}(T)$ is not bounded, then there is a sequence $\left\{w_{n}\right\}$ in $C^{3}[0,1]$ such that $u_{n}=T w_{n}$ satisfies

$$
\left|u_{n}\right|_{C^{3}[0,1]} \rightarrow \infty \text { as } n \rightarrow \infty:
$$

To simplify notation, in the following we denote by $|\bullet|_{i}$ the standard norm of the space $C^{i}[0,1]$. We shall see below that $\left\{\left|u_{n}\right|_{0}\right\}$ being bounded is equivalent to $\left\{\left|u_{n}\right|_{4}\right\}$ being bounded, thus we can assume from (12) that

$$
a_{n}=\left|u_{n}\right|_{0} \rightarrow \infty \text { as } n \rightarrow \infty
$$

In Problem (11), put $v_{n}=u_{n} / a_{n}$ and $f_{n}(x)=F\left(x, w_{n}(x), w_{n}^{\prime}(x), w_{n}^{\prime \prime}(x), w_{n}^{\prime \prime \prime}(x)\right)$. Since $\left\{f_{n}\right\}$ is a bounded sequence in $L^{2}[0,1]$, we may assume that $\left\{f_{n}\right\}$ is weakly convergent to some $f_{0} \in L^{2}[0,1]$. Then from

$$
\int_{0}^{1} a(x) h(x) d x \leqslant \int_{0}^{1} f_{n}(x) h(x) d x \leqslant \int_{0}^{1} b(x) h(x) d x
$$

for all $h \in L^{\infty}[0,1]$ with $h(x) \geqslant 0$ a.e., we see that

$$
a(x) \leqslant f_{0}(x) \leqslant b(x), \text { a.e. on }[0,1]
$$

On the other hand, since $\left\{v_{n}\right\}$ satisfies

$$
\begin{aligned}
d^{4} v_{n} / d x^{4}-f_{n}(x) v_{n} & =G\left(x, w_{n}, w_{n}^{\prime}, w_{n}^{\prime \prime}, w_{n}^{\prime \prime \prime}\right) / a_{n}, \quad 0<x<1 \\
v_{n}(0)=y_{0} / a_{n}, \quad v_{n}(1) & =y_{1} / a_{n}, \quad v_{n}^{\prime \prime}(0)=\bar{y}_{0} / a_{n}, \quad v_{n}^{\prime \prime}(1)=\tilde{y}_{1} / a_{n},
\end{aligned}
$$

therefore $\left\{d^{4} v_{n} / d x^{4}\right\}$ is a bounded sequence in $C[0,1]$. Define

$$
V_{n}=v_{n}^{\prime \prime}-\left[v_{n}^{\prime \prime}(0)+x\left(v_{n}^{\prime \prime}(1)-v_{n}^{\prime \prime}(0)\right)\right]
$$


Then $\left\{V_{n}^{\prime \prime}\right\}$ is a bounded sequence in $C[0,1]$ and $V_{n}(0)=V_{n}(1)=0$. The mean value theorem says that there is a point $\bar{x}_{n} \in[0,1]$ such that $V_{n}^{\prime}\left(\bar{x}_{n}\right)=0$. Hence the formula

$$
V_{n}^{\prime}(x)=\int_{\tilde{x}_{n}}^{x} V_{n}^{\prime \prime}(s) d s
$$

implies that $\left\{V_{n}^{\prime}\right\}$ is a bounded sequence in $C[0,1]$. Moreover,

$$
V_{n}(x)=\int_{0}^{x} V_{n}^{\prime}(s) d s
$$

gives the boundedness of $\left\{V_{n}\right\}$ in $C^{\prime}[0,1]$. This shows that $\left\{V_{n}\right\}$, and hence $\left\{v_{n}^{\prime \prime}\right\}$, is a bounded sequence in $C^{2}[0,1]$. A similar argument proves that $\left\{v_{n}\right\}$ is a bounded sequence in $C^{2}[0,1]$. Consequently $\left\{v_{n}\right\}$ is a bounded sequence in $C^{4}[0,1]$. Now by the compact embedding $C^{4}[0,1] \rightarrow C^{3}[0,1]$ we can assume for convenience that $v_{n} \rightarrow v_{0}$ in $C^{3}[0,1]$ for some $v_{0} \in C^{3}[0,1]$. Finally, letting $n \rightarrow \infty$ in (15) we easily conclude that $v_{0}^{\prime \prime \prime}(x)$ is absolutely continuous and $v_{0}$ satisfies.

$$
\begin{gathered}
d^{4} v_{0} / d x^{4}-f_{0}(x) v_{0}=0, \text { a.e. on }[0,1] \\
v_{0}(0)=v_{0}(1)=v_{0}^{\prime \prime}(0)=v_{0}^{\prime \prime}(1)=0
\end{gathered}
$$

We readily verify as was done in Section 2 that $v_{0}=0$. This contradicts the fact that $\left|v_{0}\right|_{0}=1$ since $v_{n} \rightarrow v_{0}$ in $C^{3}[0,1]$ and $\left|v_{n}\right|_{0}=1, n=1,2, \ldots$.

To use the Schauder fixed point theorem, it remains to show that $T$ is completely continuous.

The compactness of $T$ follows from the compactness of $\operatorname{cl}(\operatorname{Im}(T))$ where we use notation $\operatorname{cl}(A)$ to denote the closure of a set $A$ in an appropriate space. Let $u_{n} \in \operatorname{Im}(T)$, then $\left\{u_{n}\right\}$ is a bounded sequence in $C^{3}[0,1]$. Assume $u_{n}=T w_{n}$, $f_{n}=F\left(x, w_{n}, w_{n}^{\prime}, w_{n}^{\prime \prime}, w_{n}^{\prime \prime \prime}\right)$ and $g_{n}=G\left(x, w_{n}, w_{n}^{\prime}, w_{n}^{\prime \prime}, w_{n}^{\prime \prime \prime}\right)$. Then in Problem (11) the boundedness of $\left\{f_{n}\right\}$ and $\left\{g_{n}\right\}$ in $C[0,1]$ implies the boundedness of $\left\{d^{4} u_{n} / d x^{4}\right\}$ in $C[0,1]$. Therefore $\left\{u_{n}\right\}$ is a bounded sequence in $C^{4}[0,1]$. Again using the compact emberding $C^{4}[0,1] \rightarrow C^{3}[0,1]$ we conclude that there is a subsequence of $\left\{u_{n}\right\}$ which converges in $C^{3}[0,1]$. Hence $\operatorname{cl}(\operatorname{Im}(T))$ is compact.

Continuity follows from the fact that $w_{n} \rightarrow w_{0}$ in $C^{3}[0,1]$ implies that $u_{n}=$ $T w_{n} \rightarrow u_{0}=T w_{0}$ in $C^{3}[0,1]$. We shall argue by contradiction.

Suppose that $\left|u_{n}-u_{0}\right|_{3} \nrightarrow 0$. Then by going to a subsequence if necessary, we may assume that $\left|u_{n}-u_{0}\right|_{3} \geqslant c>0, n=1,2, \ldots$, for some constant $c$. The compactness of $T$ says that there is a subsequence, which we still denote by $\left\{u_{n}\right\}$ for convenience, such that $u_{n} \rightarrow v_{0}$ in $C^{3}[0,1]$. Noting that $w_{n} \rightarrow w_{0}$ in $C^{3}[0,1]$ in the following

$$
\begin{gathered}
d^{4} u_{n} / d x^{4}-F\left(x, w_{n}, w_{n}^{\prime}, w_{n}^{\prime \prime}, w_{n}^{\prime \prime \prime}\right) u_{n}=G\left(x, w_{n}, w_{n}^{\prime}, w_{n}^{\prime \prime}, w_{n}^{\prime \prime \prime}\right), \quad 0<x<1, \\
u_{n}(0)=y_{0}, \quad u_{n}(1)=y_{1}, \quad u_{n}^{\prime \prime}(0)=\bar{y}_{0}, \quad u_{n}^{\prime \prime}(1)=\bar{y}_{1},
\end{gathered}
$$


we see immediately that $v_{0} \in C^{4}[0,1]$ and $v_{0}=T w_{0}$. But Theorem 1 says that $v_{0}=u_{0}$, thus giving a contradiction.

Now we know that $T: C^{3}[0,1] \rightarrow C^{3}[0,1]$ is completely continuous and $\operatorname{Im}(T)$ is bounded. Let $M>0$ be large so that

$$
\operatorname{Im}(T) \subset B=\left\{u \in C^{3}[0,1]:|u|_{3} \leqslant M\right\}
$$

Then $T$ sends $B$ into $B$, so $T$ has at least one fixed point $y \in B$ by the Schauder fixed point theorem. This $y$ is a solution to Problem 2.

\section{REFERENCES}

[1] A.R. Aftabizadeh, 'Existence and uniqueness theorems for fourth-order boundary value problems', J. Math. Anal. Appl. 116 (1986), 415-426.

[2] D. Gilbarg and N.S. Trudinger, Elliptic Partial Diffenential Equations of Second Order (SpringerVerlag, Berlin, Heidelberg, New York, 1977).

[3] M.N. Nkashama and M. Willem, 'Periodic solutions of the boundary value problem for the nonlinear heat equation', Bull. Auatral. Math. Soc. 29 (1984), 99-110.

[4] R. A. Usmani, 'A uniqueness theorem for a boundary value problem', Proc. Amer. Math. Soc. 77 (1979), 329-335.

[5] Y. Yang, 'Fredholm alternatives for a fourth-order boundary value problem', submitted.

Department of Mathematics

University of Massachusetts Amherst MA 01003

United States of America 